\title{
Anthony Feneuil, Bergson. Mystique et philosophie
}

Paris, Presses universitaires de France, coll. «Philosophies », 2011, 184 p.

Daniel Vidal

\section{(2) OpenEdition}

Journals

Édition électronique

URL : http://journals.openedition.org/assr/25473

DOI : $10.4000 /$ assr.25473

ISSN : 1777-5825

Éditeur

Éditions de l'EHESS

Édition imprimée

Date de publication : 30 décembre 2013

Pagination : 189

ISSN : 0335-5985

Référence électronique

Daniel Vidal, «Anthony Feneuil, Bergson. Mystique et philosophie », Archives de sciences sociales des religions [En ligne], 164 | 2013, mis en ligne le 21 février 2014, consulté le 10 décembre 2020. URL : http://journals.openedition.org/assr/25473; DOI : https://doi.org/10.4000/assr.25473

Ce document a été généré automatiquement le 10 décembre 2020.

(C) Archives de sciences sociales des religions 


\section{Anthony Feneuil, Bergson. Mystique et philosophie}

Paris, Presses universitaires de France, coll. « Philosophies », 2011, 184 p.

Daniel Vidal

\section{RÉFÉRENCE}

Anthony Feneuil, Bergson. Mystique et philosophie, Paris, Presses universitaires de France, coll. « Philosophies », 2011, 184 p. 
1 Si la mystique est bien, selon l'expression d'Anthony Feneuil, cette expérience singulière "d'union à Dieu » - ou, pour demeurer au plus près de ce qui s'y joue et s'y avoue, le chemin en tant que tel, cette épreuve de l'impossible atteinte de Dieu -, l'inscription de cette expérience dans le champ de la philosophie comme "procédé de recherche philosophique" ne peut manquer d'entraîner une reconfiguration en profondeur des concepts propres à ce champ. Parce que son œuvre se présente comme une métaphysique de l'immanence ouverte à la transcendance, et qu'il conçoit la connaissance comme " un contact et même une coïncidence », Bergson est sans doute le philosophe qui, introduisant la mystique au cœur de sa métaphysique, a le plus radicalement mis en jeu sa pensée propre. De l'Essai sur les

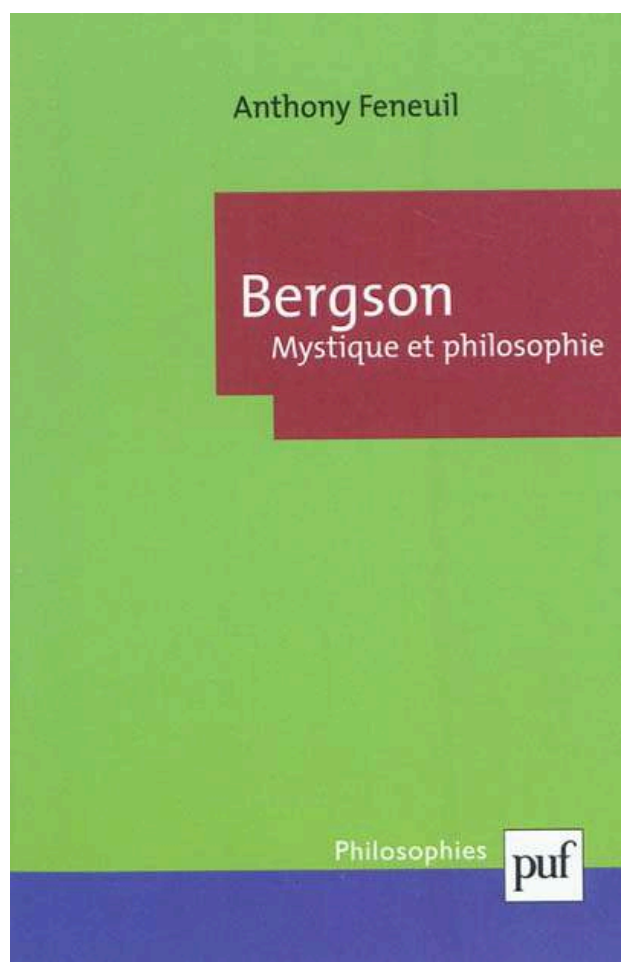
données immédiates de la conscience (1889) aux Deux sources de la morale et de la religion (1932), en passant par L'évolution créatrice (1907), A. Feneuil analyse l'approfondissement de la philosophie bergsonienne par la mise en place des catégories fondamentales permettant de poser la mystique comme noyau d'une nouvelle conceptualité philosophique. Si la philosophie se veut en mesure "d'approcher la nature de Dieu», c'est à condition de «mettre en formule» la mystique, ou, plus exactement, de repérer, dans l'énonciation mystique, ce qui se livre comme formule - et concept - de ce qui a nom « Dieu ». On sait, à s'en tenir aux auteurs mystiques du XVII ${ }^{\mathrm{e}}$ siècle (Malaval, Guilloré, Piny, Cyprien de la Nativité), mais aussi bien aux grands énoncés rhéno-flamands (Maître Eckhart, Nicolas de Cuse) que formule ou concept ne sont pas ici pris au hasard, mais fondent la mystique comme science de Dieu. De la mystique comme «nu regard sur l'objet [Dieu]", à la «science du ciel» menant à Dieu comme «concept universel " peut déjà se définir un espace de confrontation/traduction qui autorise le déploiement de la question de Dieu en argument philosophique. Car la formule en mystique a bien cette fonction de resserrer l'énoncé à sa plus simple expression, à sa « phrase » la plus condensée, qui peut alors s'inscrire « dans un réseau de concepts à développement potentiellement infini ». Ainsi de l'intuition, concept central du bergsonisme, qualifiant ici la raison mystique, qui, en travail au sein de l'argumentaire philosophique, ouvre radicalement le système conceptuel. «Vision directe de l'esprit par l'esprit », selon la définition de Bergson, l'intuition est cette «saisie directe et immédiate de l'absolu ». Si l'auteur ne privilégie pas ces références, du moins dispose-t-il l'émotion, cet autre concept central du philosophe, comme constitutive de la notion de "personne », en tant que la personne ainsi instituée ouvre l'analyse à ce qui fonde la relation à autrui, condition de toute pensée de religion. Intuition, émotion, personne : voilà le triangle à partir duquel il est possible de dégager, chez Bergson, une «théorie » de la religion adossée à son impératif mystique. 
2 Si Bergson conçoit la personne comme émotion, ce n'est pas au sens convenu de l'effervescence des religions dites "émotionnelles ». C'est pour rendre compte à la fois des "dieux personnels ", qui, dans les religions "statiques » où tout événement est référé à une intentionnalité, personnifient cette causalité sur le mode d'un «anthropomorphisme grossier» - et pour indiquer que cette fonction fabulatrice participe également de la théorie intellectualiste de la personne, qui implique, selon A. Feneuil, « une conception de Dieu comme unité absolue, c'est-à-dire absolument indépendante.» Tel est «le Dieu des philosophes». Pour échapper à cette double limitation, il faut reprendre à nouveau frais la question de la personne, et, propose l'auteur, de concevoir la personnalité, «l'unité personnelle », comme achèvement et dépassement « $\mathrm{du}$ processus d'individuation». Si la personnalité "émane de l'émotion ", à l'émotion profonde répond la personne en sa "totalité ». Ainsi vient le moment mystique au cœur de la philosophie. Car telle émotion projette la personne en un point « hors de plan », après qu'elle l'a déplacée hors de soi. Bergson dit "extase » cette capacité à se libérer des déterminismes du temps et des contextes de situation. L'acte libre, écrit-il, « est celui où je suis tout entier ", et qui vaut "triomphe des déterminismes, le temps d'un instant ». Si l'auteur n'ignore pas cette problématique de l'instant, du moins est-il acquis que cette émotion « supra-intellectuelle » ne décide pas d'un régime de représentation, mais, pourrait-on dire, de pure présence, qui constitue, dans la pensée de Bergson l'accomplissement de ce qu'il nomme effort, cet élan vital en son inachèvement même. Comme il en va de « l'œil pour faire apparaître une étoile qui aussitôt rentre dans la nuit ». Une telle émotion, ajoute-t-il, "ressemble sans doute, quoi que de loin, au sublime amour qui est pour le mystique l'essence même de Dieu ». L'extase mystique se dit excès, par quoi s'atteint, et se définit, le divin. Point ultime de l'émotion, qui, précise Feneuil, "n'est ni hors du temps, ni inconnaissable », mais qui "s'éprouve dans le temps ", - dans l'instant, on l'a vu, mais dans l'élan de toutes les durées antérieures. Toute une vie intime est requise pour telle expérience. Aussi bien l'extase est-elle "un acte de création continue de soi ", et l'émotion qui en est à l'origine peut être dite « impulsion de cet acte ».

3 Le moment mystique ne s'entend, dans la philosophie de Bergson, que comme modalité exceptionnelle - et nécessaire - de l'élan. Mais celui-ci ne s'accomplit jamais pleinement en l'homme. Plus exactement, la personne humaine ne peut être "effort pur de création ", puisque toujours "en manque d'elle-même ", et toujours reprise "d'actes déjà faits" - donc prise en une histoire singulière et collective, et donc toujours " autre » qu'elle-même et multiple. La conclusion s'impose : "Dieu seul est parfaitement lui-même ", en ce que, "acte de création ", il est distinct de la création elle-même. Une émotion pure, ce "pur jaillissement ", "s'épanouirait en idée si elle voulait, âme toute pure, se donner un corps ", écrit Bergson. Dieu? «Une personne coïncidant avec une émotion », propose Anthony Feneuil, cet élan lui-même s'accomplissant à l'infini. Seul, alors, un « homme privilégié » peut connaître ce qui est incommensurable, et, proprement, indicible. Tel est le mystique, en réalité "plusqu'homme ", qui, parce que tout entier habité d'enthousiasme, est pur dépassement des frontières du «bien-être ", de soi et d'autrui, et immédiatement « communicatif ». De ce soulèvement de profondeur, de cette émotion transfigurante, irradient en effet des "résonances sentimentales », qui circulent dans le réseau collectif. Nietzsche nommait ce Sujet «surhomme », que M. Cacciari suggérait, dans Le Jésus de Nietzsche, d'appeler " outre-homme », à quoi sans doute eut consenti Bergson. Mystique est ainsi celui qui 
brise l'ordre de la nécessité et "reprend la marche en avant », cet élan qui pour finir est l'autre nom de Dieu. Dont le premier est amour.

L'enthousiasme est cette émotion qui appelle l'individu à sortir de soi, et faire aussitôt scandale au monde, brisés tous les cadres de l'expérience humaine. Feneuil précise qu'ainsi le mystique ne devient pas autre, mais, parce que dépassant les déterminismes et frontières qui limitent sa liberté, il fait retour dans le «réel» en tant que «son propre créateur ». On comprend mieux par là cette difficulté du « retour» que disait Thérèse d'Avila. Cette capacité à se produire sujet pleinement de soi répond à l'amour divin comme « effort de création tenu en sa pureté ». Du mystique à Dieu, une relation s'établit entre une pure oblation/ablation de soi, et une pureté d'amour, cette " unicité absolue ", cette "extase " à l'infini. Et de même que, pour reprendre la formule de l'auteur, "l'existence d'un mystique rend toute l'humanité mystique", de même l'amour divin est appel à l'universel, impératif catégorique autant que pure donation. D'une émotion mystique, écrit Bergson, «quelque chose y répond au fond de notre âme ». De Dieu comme amour, une "relation de réciprocité » émane immédiatement. C'est dire qu'entre une expérience mystique et Dieu comme "continuité de jaillissement » se fonde la " participation à l'être divin », et se définit, selon Bergson, le seul espace possible de la connaissance de Dieu.

5 C'est dire aussi qu'il n'est pas, pour le philosophe, de transcendance, mais un « passage à la limite au sein du même ordre des choses ", qui permet d'aller « encore au-delà ", au principe même de l'élan vital. La vie, écrit Bergson, «vise essentiellement à capter de l'énergie utilisable pour la dépenser en action explosive». De la personne privilégiée qui s'accomplit dans l'expérience de Dieu, à Dieu, cet «amour» d'impossible expérience, le jeu se joue à la limite, en un point « hors du plan » qui ne se définit que par le plan lui-même. Aussi bien l'expérience mystique permet-elle de «repérer dans l'immanent la marque du transcendant ", et de nouer radicalement celui-ci à celui-là. C'est pourquoi le philosophe, selon l'auteur, ayant «ressenti l'enthousiasme du mystique, peut prétendre théoriser cette expérience et connaître Dieu ». Sans pour autant, note-t-il, prendre en compte la question du Christ, sa divinité, son humanité. Bergson à Jean Guitton: «Du point de vue philosophique et méthodologique où je me plaçais, cette existence historique [du Christ] pouvait apparaître comme momentanément secondaire ». Plus encore : Anthony Feneuil ne relève chez Bergson que la conception d'un Christ "étonnamment désincarné ». Il apparaît cependant que la question mystique et la question de Dieu, chez Bergson, ne renvoient pas, ni immédiatement, ni par inclination, à la seule et incontournable conception chrétienne, mais se déploient plus à l'écart, tout privilège aboli, toute captation dogmatique récusée (ainsi du dogme de l'incarnation). L'émotion comme raison et marque de religion, ou, pour reprendre la terminologie de H. Bremond, le «sentiment », comme paradigme de religion, ne sont pas affaire de théologie. 